\title{
Exploring Alternative Flexible OpenCL (FlexCL) Core Designs in FPGA-based MPSoC Systems
}

\author{
Dan Connors \\ Department of Electrical Engineering \\ University of Colorado Denver \\ Denver, Colorado \\ Dan.Connors@ucdenver.edu
}

\author{
Eric Grover \\ Department of Electrical Engineering \\ University of Colorado Denver \\ Denver, Colorado \\ Eric.Grover@ucdenver.edu
}

\author{
Blake Caldwell \\ Department of Electrical \& Computer \\ Engineering \\ University of Colorado Boulder \\ Boulder, Colorado \\ Blake.Caldwell@colorado.edu
}

\begin{abstract}
Open Compute Language (OpenCL) has been proposed as a platform-independent parallel execution framework to target multicores, graphics processing units (GPUs), digital signal processors (DSPs), and other custom accelerators. Traditionally OpenCL is designed to scale across system generations by having each new model increase the available resources: processing cores, register file entries, and shared (localized) physical memory. However, when evaluating all potential models capable of running OpenCL the numerous performance trade-offs associated with these competing resources makes for a large exploration space. Specifically there are advantages to systematically analyzing the OpenCL parallel expressions to eliminate processor elements that constrain the overall design. We have designed an analysis tool for evaluating OpenCL kernels and the potential processor configurations in FPGA-based multiprocessor systems-on-chip (MPSoC) designs. The Flexible OpenCL (FlexCL) system determines integrated hardwaresoftware customization opportunities related to processor design across sets of OpenCL applications. The paper provides an evaluation of various OpenCL components synthesized to FPGAbased multicore design alternatives.
\end{abstract}

\section{INTRODUCTION}

General-purpose computing on graphics processing units (GPGPU) makes use of a graphics-processing unit (GPU), traditionally purposed only for computer graphics. In the past few years, there has been an accelerated adoption of GPGPU parallel computing techniques for the high-performance computing and embedded domains. Open Compute Language (OpenCL) [1] is a platform-independent parallel execution framework able to scale across multicore models (CPU, GPU, DSP, and accelerators) of perhaps varying hardware generations, and heterogeneous platforms. The core utility of OpenCL model is the promotion of a virtual execution model that can be used to target numerous alternative designs with various differentiated resources.

Over the same few years, Field Programmable Gate Array (FPGA) technologies have likewise evolved. Currently highperformance FPGAs have scaled to the point of including dedicated processors [2], DSPs, multipliers, and memories with customizable logic on the same chip. In many application

Permission to make digital or hard copies of all or part of this work for personal or classroom use is granted without fee provided that copies are not made or distributed for profit or commercial advantage and that copies bear this notice and the full citation on the first page. To copy otherwise, or republish, to post on servers or to redistribute to lists, requires prior specific permission and/or a fee.

RAPIDO '13, January 21 2013, Berlin, Germany

Copyright 2013 ACM 978-1-4503-1539-5/13/01 ...\$15.00. domains, the hybrid (CPU+FPGA) may have significant performance advantages over single core and even multicore CPU hardware architectures [4]. While an FPGA-based design may offer higher performance at lower power than multicore CPU and DSP systems, they still require significant time-to-market in terms of design and validation. At the same time, FPGAs serve as extremely effective targets in prototyping frameworks, allowing the design space [5], [6] of performance, power, cost, space, and reliability trade-offs to be considered for domains of interest.

As OpenCL execution and runtime models enable a software expression of an algorithm's parallelism to scale to available resources, there is potential for a monumental shift in the embedded design field. At some level, the software kernels describe the same concepts that custom designers include as parallel hardware circuits and processing elements cooperating to execute a specific computation task. There are several potential advantages to the microprocessor design process in mapping the concepts within the OpenCL model to FPGA custom designs. First concerning design time, there is a steady proliferation of OpenCL software kernels of algorithms spanning numerous domains that are ready to be shared with open source communities. Second, there are opportunities to take advantage of software optimization (compilers) to improve the targeting of the design space. Similarly, by taking into account OpenCL concepts, a refined implementation can be described within that same design space. Finally, for a set of algorithms, there is potential to balance the requirements carried out in software implementation rather than including dedicated hardware for any one kernel.

Our work explores customizable OpenCL multicore processors. We use a flexible OpenCL (FlexCL) core description to rapidly generate customized processors for both specific application and many application cases. A highlight of the FlexCL framework is that the OpenCL kernel requirements reduce the need to include a full-scale processor core. An analysis can deliver the minimum set of processor features (operation codes, registers, pipeline features, floating-point hardware, and memory resources), and a minimum set of OpenCL features (special-purpose registers, local memory, and synchronization). Our framework is based on the MB-Lite soft-processor (open-source Xilinx Microblaze implementation) and we discuss FPGA synthesis outcomes for variations on design parameters. In this way the FlexCL framework can provide the potential of OpenCL-enabled FPGA-based MPSoC designs.

The paper includes the following sections. Section 2 provides background on the OpenCL computation model and motivation for customized OpenCL designs in heterogeneous systems. Section 3 illustrates the FlexCL approach. Section 4 includes initial experimental results, and Section 5 includes a summary and future work. 


\section{BACKGROUND AND MOTIVATION \\ 2.1 Related Work}

Much of the other related work being done is using an OpenCLto-gates approach. FlexCL is doing OpenCL-to-native machine code, which is then loaded onto the FPGA at runtime and executed. This has the advantage that the algorithm to be run can be changed without needing to generate a new bit-stream for the FPGA. Altera's OpenCL toolset was recently announced [15]. Starting with OpenCL as an input, the the toolset generates the host executable and the associated FPGA bit stream. The MARC system [16] uses templates of an architecture that is customized at the HDL level through analysis of OpenCL kernel code. RAMPSoC is a framework for generating an MPSoC composed of multiple microprocessors and specialized hardware accelerators for the algorithm being executed [17]. A method of dynamic runtime partial reconfiguration for adding and removing blocks is also demonstrated. The language front-end is $\mathrm{C} / \mathrm{C}++$. Since each processor is a full-function processor each one requires more resources than a compute unit in FlexCL.

\subsection{Open Compute Language (OpenCL)}

The OpenCL language is just one method of describing dataparallel algorithms. The Nvidia Compute Unified Device Architecture (CUDA) [3] is another data-level parallel programming model, established in 2007, to target the off-loading of general-purpose computations on Nvidia GPUs. While similar, OpenCL is implemented as a platform-independent framework capable of targeting heterogeneous processors. OpenCL applications consist of two components: (1) a host program directing management of one of more compute devices using a series of application programming interfaces (API) calls, and (2) a kernel program executing on the compute devices. For the purpose of this paper, it is only necessary to review the concept of the OpenCL kernel.

OpenCL kernels are written in standard C code with annotations to specify parallelism and explicitly controlled memory structures and thread interactions. Figure 1 illustrates a common example of an OpenCL kernel for averaging three neighboring input values to generate an output value. Within OpenCL, this kernel explains the independent work performed in parallel by potentially any number of parallel hardware threads.

$$
\begin{aligned}
& \text { kernel void Filter(_ global float *In, _ global float *Out) } \\
& \text { //Get the index of the current element to be processed } \\
& \text { int id = get_global_id }(0) \text {; } \\
& \text { //Computation performed by each workitem } \\
& \text { Out }[\mathrm{id}]=(\operatorname{In}[\mathrm{id}-1]+\operatorname{In}[\mathrm{id}]+\operatorname{In}[\mathrm{id}+1]) / 3 \text {; }
\end{aligned}
$$

\section{Figure 1 Example OpenCL Kernel (3-Cell Average Filter)}

A programmer designates an OpenCL kernel to be launched on a compute device as an N-dimensional workspace. Each workspace is divided into smaller $\mathrm{N}$-dimensional workgroups that are scheduled on independent compute units within the compute device. Each workgroup consists of a number of workitems that are directly mapped to hardware threads (contexts) and execute on processing elements. Each workitem has a unique ID that is accessible from the kernel using OpenCL builtins such as get_global_id(0). The IDs are used to distinguish the data to be accessed (read or written) by each workitem. Workgroups are described within OpenCL to allow (1) efficient mapping of data- parallel tasks to parallel hardware (compute units) and (2) communication between workitems, specifically on a designated compute unit with a dedicated physical memory (local memory).

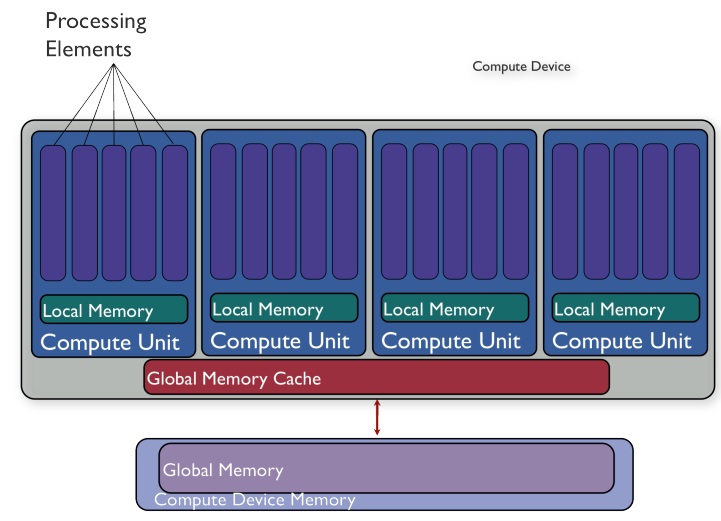

Figure 2 Overview of OpenCL Architecture Model (Compute Device, Compute Unit, Processing Element)

Figure 2 represents the standard OpenCL execution model of a target compute device. In this view, a compute device must include 1 or more compute units and each compute unit must have 1 or more processing elements (on which threads carry out the computation of described workitems). The overall design of the OpenCL architecture allows a large number of workgroups to be simultaneously scheduled by the runtime system of the compute device, which is generally a GPU target. Each workgroup carries out the same overall kernel computation, but has a designated subset of the input and output space. However, there are a few key execution issues when workitems within the same workgroup execute on different paths. These issues relate directly to the design space of customizable OpenCL-enabled cores, and are described in the following three subsections.

\subsubsection{Workitem Model \& Workgroup Coordination}

The expression of parallelism in OpenCL is implicitly constructed in kernels by describing the workitem execution using a set of built-in references that coordinate and divide the entire execution space. Figure 3 shows the OpenCL description of a 2-dimensional workspace subdivided into the workgroups and workitem coordinate system.

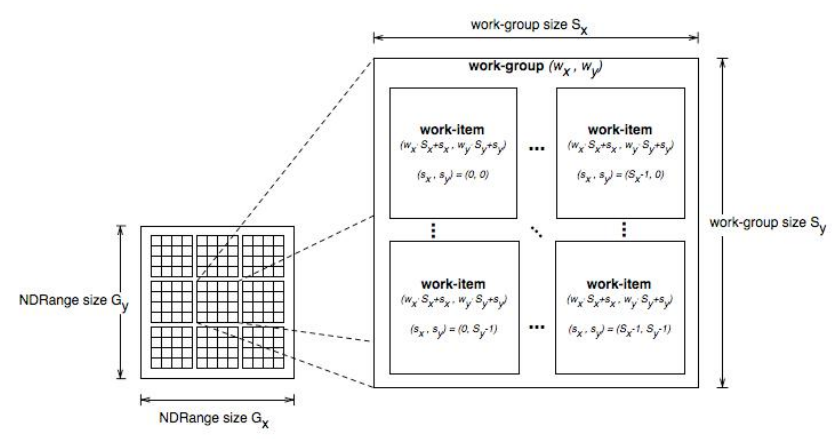

Figure 3 OpenCL Workspace: 2-D Example

The OpenCL built-ins serve as programming-level placeholders that get transformed into per-thread values when workitems are mapped to hardware thread contexts in compute units. There are three categories: Global, Local, and Individual. Each takes on a dimension descriptor respectively reserved for dimensions 0,1 , and 2 (mapping to $\mathrm{x}, \mathrm{y}$, and $\mathrm{z}$.) 
- get_local_size (dimidx) - returns the number of local work-items in the work-group specified by dimension

- get_global_id (dimidx) - returns the unique global work-item ID for the specified dimension

- get_local_id (dimidx) - returns the unique local workitem ID in the work-group for the specified dimension

- $\quad$ get_num_groups (dimidx) - returns the number of workgroups for the specified dimension

- $\quad$ get_group_id (dimidx) - returns the unique ID of the work-group being processed by the kernel

- get_work_dim (dimidx) - returns the number of dimensions of the data problem space

- get_global_size $(\operatorname{dimidx})$ - returns the number total work-items for the specified dimension

In Figure 3, the two-dimensional example, a workitem can determine its location in a 2D array by using get_global_id(0) and get_global_id(1) when geometric positions of $\mathrm{x}$ and $\mathrm{y}$ are mapped to the $2 \mathrm{D}$ array. Workitem local offsets are referenced using get_local_id(0) and get_local_id(1).

Table1: OpenCL Intrinsic: Global, Local, Individual

\begin{tabular}{|l|l|}
\hline Execution Scope & OpenCL Intrinsic \\
\hline $\begin{array}{l}\text { Global (Kernel } \\
\text { workspace) }\end{array}$ & $\begin{array}{l}\text { get_local_size() } \\
\text { get_work_dim() } \\
\text { get_global_size() } \\
\text { get_num_groups() }\end{array}$ \\
\hline Local (workgroup) & get_local_id() \\
\hline $\begin{array}{l}\text { Individual } \\
\text { (workitem) }\end{array}$ & $\begin{array}{l}\text { get_local_id() } \\
\text { get_global_id() }\end{array}$ \\
\hline
\end{tabular}

Since most processor implementations provide OpenCL by way of a system library, there are not dedicated machine hardware resources specialized for OpenCL implementations. In generic processors, the built-ins are typically assigned to registers or assigned to memory locations (that must be accessed from global device memory). Table 1 shows the breakdown of the built-in terms as can be shared among the workspace (Global), and workgroup (Local), and those terms that have unique values per workitem (Individual). By analyzing each OpenCL kernel's requirements, FlexCL cores can integrate the built-in elements into the system design and diminish the required hardware space.

Similar to the built-in elements, the primary scaling of resources of data-parallel architectures and GPU designs is achieved by coordinating multiple workitems to execute on multiple processing elements by using common facilities such as fetch and decode stages. Figure 4 shows a traditional OpenCL hardware design of a compute unit, in which multiple workitems of a workgroup have been assigned resources from the large computeunit pools of (1) registers, (2) process/thread content. The coordination of the system takes care dispatching the available workitems from the set of workgroup contexts. As shown, the shared register file is a set of resources divided among the groups of thread contexts. Designs may provide a limit on the number of thread contexts as well as be limited by the use of resources by each thread context. For example, if each thread context is assigned $\mathrm{X}$ registers, then the register file may become a limiting factor as it must provide equal resources to each thread.

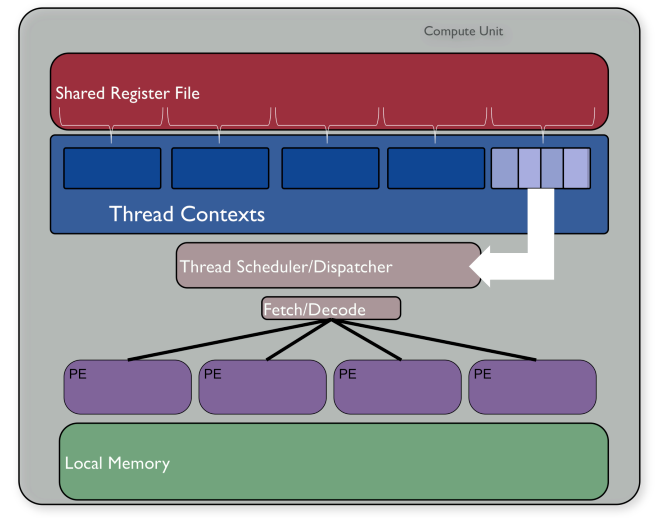

\section{Figure 4 Traditional Construction of OpenCL Workgroup Context Execution}

Motivation: In the FlexCL exploration framework, one goal is to relate the available resource trade-offs between traditional multiprocessor and OpenCL processor designs. For multiprocessor designs, one measurement of scalable parallelism is based on the number of processing elements on chip. However, as OpenCL-based GPGPU computations are based on massively multithreading techniques, a more accurate level of a design's parallelism includes the number of available thread contexts (and registers available to those contexts) per compute unit. As such, any FlexCL customization (reduced operation codes, registers, etc) can be translated into space for more thread contexts on each compute unit.

\subsubsection{Workitem Divergence}

In the Nvidia CUDA terminology, divergence issues for threads (OpenCL's workitems) in blocks (OpenCL's workgroups) are referred to as branch divergence [11]. Branch divergence can result in significant execution overhead, as hardware that is typically highly utilized to a full set of threads within a compute unit becomes idle. Typically branch divergence can be the result of data-dependent conditions or when the workitem IDs are used to isolate a specific set of workitems from the rest of the workgroup. Figure 5 illustrates an example of a branch divergence where the branch condition for an individual workitem depends on independent data values. Programmers can sometimes statically restructure their code to eliminate workitem-based branches with divergence. However true data-dependences are difficult to resolve. With respect to FlexCL processing, branch divergence is an important characteristic to address since the goal is to break the strict execution model in which all workgroups execute the identically composed kernel.

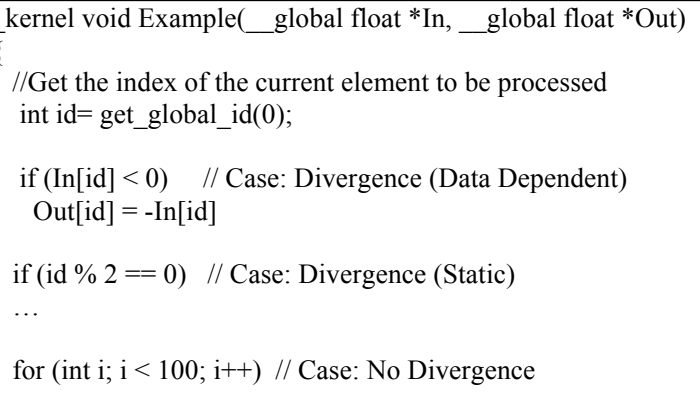

Figure 5 OpenCL Workgroup Example Branch Divergence 
Figure 6 shows the necessary hardware support for the case of branch divergence taken in most OpenCL-based GPU architectures. In Figure 6(a) scenario, four workitems of the same workgroup are scheduled to execute a control sequence involving four basic blocks. For the case when workitems $\mathrm{WG}_{0}$ executes taken, and the other workitems $\mathrm{WG}_{1}, \mathrm{WG}_{2}, \mathrm{WG}_{3}$ execute nontaken, there is branch divergence. As workgroups are dispatched to execute identical instructions (common fetch, decode, and execute stages), the typical approach is to use forms of predicated execution [13] shown in Figure 6(b). In this case, special hardware support is used to allow each individual instruction to use predicate mask registers to conditionally nullify instructions. The benefits of the approach are that the instructions maintain the same instruction sequence point, while the deficiency is that the hardware must provide such support. In the case where FlexCL performs customizations for each OpenCL kernel, oftentimes there are ways to analyze the code to determine whether branch divergence is data-dependent or workgroup-dependent.

Motivation: FlexCL kernel analysis can reveal branch behaviors (data-dependent versus workgroup-dependent) to synthesize distinct compute units with and without branch divergence support. In these cases, kernel-specialization provides benefits of saving hardware resources in the design space.

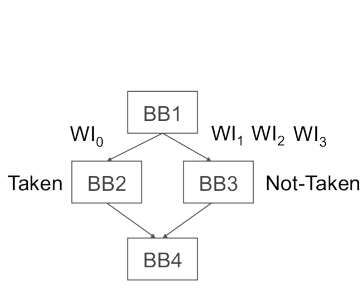

(a)

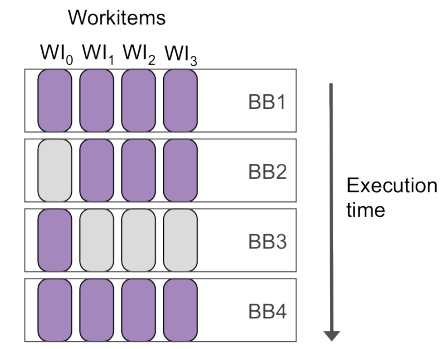

(b)
Figure 6 OpenCL Divergence (a) and Hardware Support for Workitem Predicated Execution (b)

\subsubsection{Workgroup Synchronization}

OpenCL workgroups are not able to synchronize globally with other workgroups. Instead, only workitems of the same workgroup can synchronize at programmer-based control points in kernels. Intra-workgroup synchronization is based on splitting large workgroups into smaller implementation-specific groups of hardware threads. In the case of Nvidia CUDA/OpenCL support for GPUs, workgroups are carried out in multiples of 32 threads collectively called a Warp [ ]. In the case of AMD OpenCL, workgroups are defined by multiples of the Wavefronts (hardware primitive of 64 threads). An example of the synchronization of workitems of a workgroup is shown in Figure 7. In this example, the programmer may define a workgroup as 512 workitems, yet the programmer may not know the platform being used to execute the code. In order for the OpenCL-enabled device to carry out the expressed parallelism, the underlying hardware may use divisions of $4,8,16,32,64$, etc. threads. In such case the divisions of a workgroup each maintain an execution point in the kernel and for a given point in time, each division could be positioned at a different execution point. To synchronize the divisions, the OpenCL barrier() programming element requires the hardware implementation to force all divisions to reach an agreement point in code before continuing execution. The example of Figure 7 shows the utility of synchronization in which multiple threads will share the contents of local memory, a shared access memory that is physically local. Workitems in the example use data values loaded by other workitems. So to preserve barrier() semantics, OpenCL coordinates execution such that all divided groups of the workgroups reach a common point before continuing on.

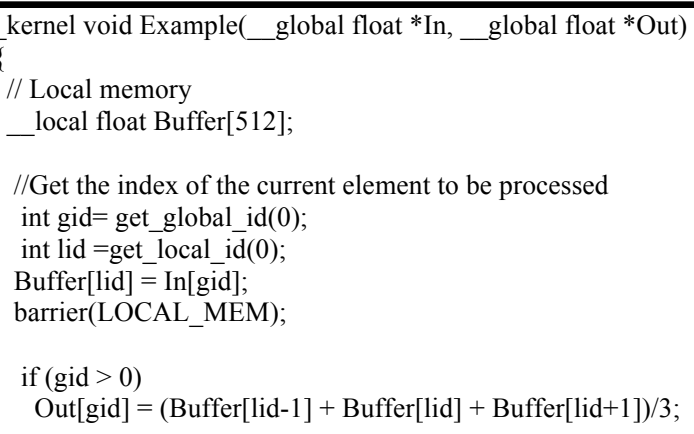

Figure 7 OpenCL Barrier() Synchronization

Motivation: An OpenCL kernel's specific use of synchronization and compute unit's local memory can substantially impact the design of the processor architecture. Kernels can be coded without the use of synchronization, alleviating the need to provide synchronization capabilities in the compute unit implementation. Similarly, synchronization may only be necessary for a subset of workgroups in the workspace (example: workgroups handling the processing of boundary cases). Therefore only a subset of compute units requires synchronization support.

\section{APPROACH}

Multi-Processor System-On-Chip (MPSoC) architectures have become widespread for many real-time application domains [4]. Such systems span a very large spectrum of design alternatives. In order to explore design constraints (e.g. energy consumption or time-to-market), as well as explore many possible configurations, there are options for exploring MPSoC designs in FPGA-based systems [8]. FPGA prototyping and emulation systems often serve as more accurate environments for exploring hardware-software trade-offs over cycle-accurate simulators.

The FlexCL architecture framework involves both OpenCL kernel analysis to reveal execution properties and a parameter-based FlexCL core. The combination of the static kernel analysis when coupled with the design space, allows a number of OpenCLenabled MPSoC architectures to be derived. Figure 8 shows an example deployment of FlexCL in which multiple distinct compute unit configurations are deployed in the hardware.

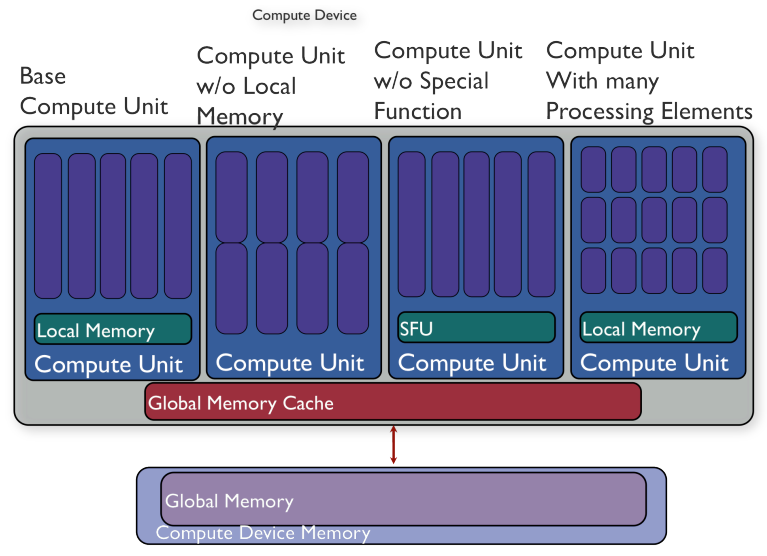

Figure 8 FlexCL Exploration: Alternative Compute Resource 


\subsection{FlexCL Core Configuration}

The FlexCL core is based on the Xilinx soft-processor MicroBlaze [9]. MicroBlaze is a 32-bit RISC-based architecture, similar to the MIPS architecture, traditionally designed for Xilinx FPGAs. As a soft-core processor, MicroBlaze is implemented in the logic fabric of Xilinx FPGAs, and there are several open source implementations (OpenFire, MB-Lite, MB-Lite+, and myBlaze). FlexCL is based on the MB-Lite MicroBlaze implementation in VHDL. The FlexCL description includes design directives to organize patterns of FlexCL processors with features enabled or disabled (i.e. building a processor with only a subset of operation codes). This allows optimization of the hardware to the algorithm being executed or performanceestimated for multiple synthesized configurations for each partition.

The following FlexCL design elements are configuration targets:

- Number of OpenCL Compute Unit (CUs)

- Number of OpenCL Processing Elements (PEs)

- Number of registers available to cores in an CU

- Opcode selection: Include/exclude instructions: integer multiply/add/subtract/compare, AND/OR/XOR, shift, sign extend, conditional/unconditional branch, barrel shift, FPU add/subtract/multiply/divide

- Local memory size and number of local memory banks

- Number of floating-point processing units (FPUs) in CU

- Other features of core: pipeline register forwarding, memory result forwarding

The FlexCL design based on Figure 9 was built and executes instructions in simulation. This design was used to obtain FPGA resource usage for many design alternatives to understand the trade-off of OpenCL customization and FPGA resources.

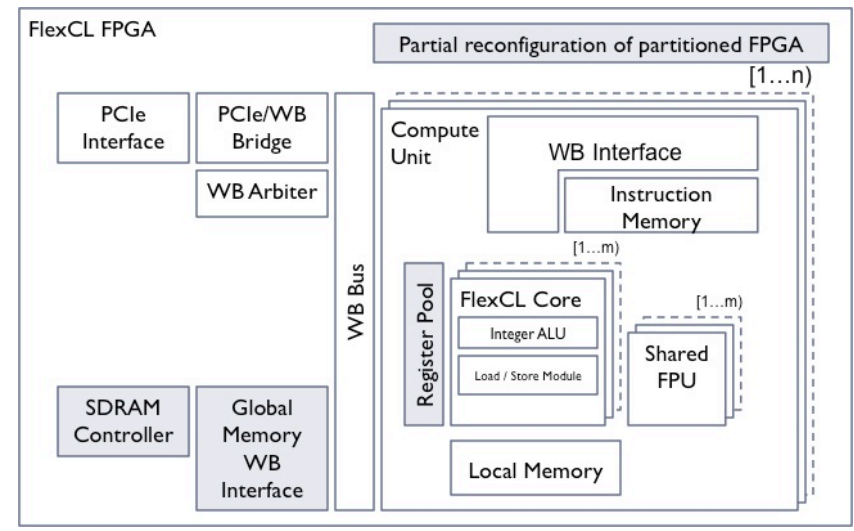

Figure 9 FlexCL MPSoC Overview

Figure 9 shows the isolation of the compute unit implemented using a Wishbone bus topology. From the perspective of FPGA routing, more efficient topologies exist that will be considered in a future revision (i.e. ring or star). Each compute unit consists of a configurable number of cores, configurable number of FPUs, and a configurable amount of local memory. Each core within the compute unit consists of a modified version of FlexCL. Each core executes in lock-step with the other cores within a compute unit, and consists of a 32-bit data path, 5-stage pipeline, and 32 registers. The lock-step model described in OpenCL for each compute unit is a primary source of real-estate savings as multiple cores are able to share the fetch and decode of operations from a common program counter location.

Floating-point units (FPUs) within a compute unit are shared between cores. The FPU consists of floating point cores generated from Xilinx CoreGen. With the exception of division, each core is fully pipelined to allow FPU sharing between cores to be very efficient given the amount of FPGA resources each FPU requires. Local memory is arranged into banks. Each bank operates independently for simultaneous read or write operations. Local addresses are coalesced across the banks. If two cores attempt to access the same bank then their operations are serialized. Block RAM is used on the FPGA for local memory. The RAM itself has a 1-cycle latency, but interconnects increase latency to 3 cycles.
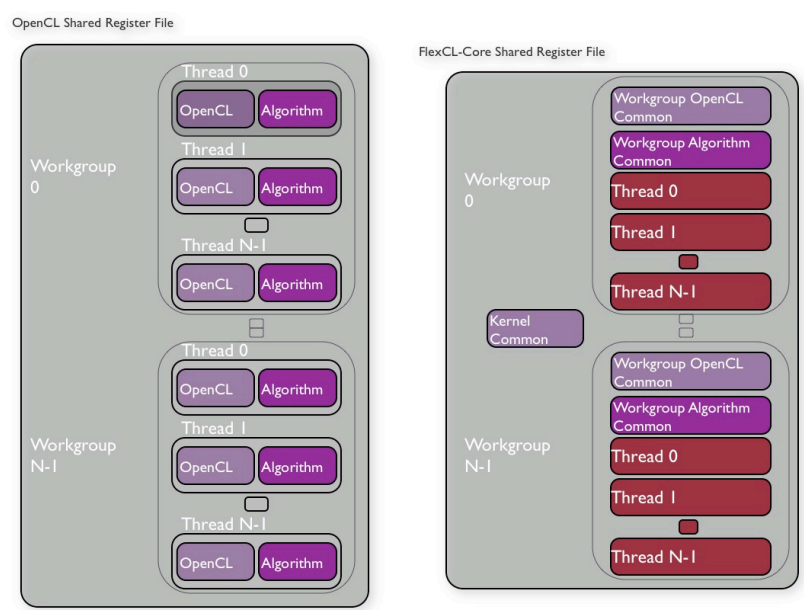

Figure 10 FlexCL Register Architecture

A central component of Figure 9 is the FlexCL core. For specific OpenCL kernels, custom cores (decode stage, ALU functions, register bypassing) can be synthesized. For example, the elimination of operation codes from the full ISA can result in space savings for soft-core implementations. As the core designs are replicated many times throughout the compute unit, there is a magnified savings potential for custom FPGA-based MPSoC.

Figure 10 shows the difference of register file implementation between a traditional GPU compute unit constructed of soft cores and the FlexCL compute unit. In the FlexCL case, the register file can be customized for each algorithm to (1) contain the minimum number of algorithm registers and (2) share common OpenCL kernel and workgroup built-in values.

\subsection{OpenCL Kernels}

The work presented here aims to expand upon the facilities available part of the LLVM compiler framework [10] for compiling C-based code to an intermediate representation and then to architecture-specific assembly instructions. The compilation of C-code to LLVM IR has been well developed and we implemented a FlexCL code generator.

The following describes the eight OpenCL kernels used for experimental analysis to determine the potential benefit of reducing required hardware logic on a per-application basis. Each benchmark is assigned a letter (A thru $\mathrm{H})$ and described:

- $\quad$ BitonicSort (A): a parallel algorithm for sorting, used as a construction method for building a sorting network. 
- FastWalshTransform (B): an efficient algorithm to compute the Walsh-Hadamard transform (WHT).

- FloydWarshall (C): a graph analysis algorithm for finding shortest paths in a weighted graph and finding transitive closure of a relation $\mathrm{R}$.

- Histogram (D): a representation showing the distribution of data.

- Mandelbrot (E): a calculation based on a mathematical set of points whose boundary is a distinctive twodimensional fractal shape.

- MatrixTranspose (F): an algorithm that efficiently computes the reflection of $\mathrm{A}$ over its main diagonal to obtain $\mathrm{A}^{\mathrm{T}}$

- RadixSort (G): a non-comparative integer sorting algorithm that sorts data with integer keys by grouping keys by the individual digits which share the same significant position and value.

- $\quad$ Reduction $(\mathrm{H})$ : the repeated application of an addition operator to a set of data values.

The base Microblaze ISA includes approximately 110 operation codes. The compilation of OpenCL kernels generates both a base version and an optimized version of code for the target FlexCL core. Table 2 shows the static operation count for each target kernel. On average, the LLVM compiler is able to reduce the number of operations for the target processors by $67 \%$. This shows a good design characteristic that software compilers are still involved in the performance process.

Table 2. Operation Counts for Targeted OpenCL Kernels

\begin{tabular}{|l|l|l|l|}
\hline OpenCL Kernel & $\begin{array}{l}\text { \#Ops } \\
(\text { Base })\end{array}$ & $\begin{array}{l}\text { \#Ops } \\
\text { (Opti) }\end{array}$ & $\begin{array}{l}\text { \%Operation } \\
\text { Reduction }\end{array}$ \\
\hline BitonicSort (A) & 382 & 116 & $69.6 \%$ \\
\hline FastWalshTransform (B) & 255 & 82 & $67.8 \%$ \\
\hline FloydWarshall (C) & 376 & 93 & $75.3 \%$ \\
\hline Histogram (D) & 458 & 148 & $67.7 \%$ \\
\hline Mandelbrot (E) & 4854 & 2822 & $41.9 \%$ \\
\hline MatrixTranspose (F) & 493 & 108 & $78.1 \%$ \\
\hline RadixSort (G) & 1062 & 297 & $72.0 \%$ \\
\hline Reduction (H) & 457 & 151 & $67.0 \%$ \\
\hline
\end{tabular}

Table 2 shows the comparison for FPGA design technique on the overall operation codes that are required by each kernel. The first column indicates the kernel $(\mathrm{A}-\mathrm{H})$, and the next two columns indicate the distinct type of operation code needed by the OpenCL processor for each kernel implementation with FlexCL processor (for base and optimized code). As shown in Table 3, the average reduction in operation set is $9.2 \%$, shown per kernel benchmark in the fourth column. Again, the use of compilers shows that there is a good potential for software techniques to modify the requirements of hardware and lead to variations in the design space. Each included operation encoding requires the decode stage to provide control signals and for the processor pipeline to have dedicated resources for implementing each operation code. In this view, the custom-OpenCL processor design. The set of kernels require a total of 33 operation codes. As illustrated by the final column, many kernels require a small set: $39.4 \%$ of that set.

Table 3. Operation Set Requirements of Eight Kernels

\begin{tabular}{|c|c|c|c|c|}
\hline Kernel & $\begin{array}{l}\text { Opcodes } \\
\text { (Base) }\end{array}$ & $\begin{array}{l}\text { Opcodes } \\
\text { (Opti) }\end{array}$ & $\begin{array}{l}\% \\
\text { Reduction }\end{array}$ & $\begin{array}{l}\text { Required \% of } \\
\text { FlexCL ISA }\end{array}$ \\
\hline A & 16 & 13 & $18.8 \%$ & $39.4 \%$ \\
\hline $\mathrm{B}$ & 19 & 19 & $0 \%$ & $57.6 \%$ \\
\hline $\mathrm{C}$ & 18 & 15 & $16.6 \%$ & $45.5 \%$ \\
\hline $\mathrm{D}$ & 16 & 13 & $18.8 \%$ & $39.4 \%$ \\
\hline $\mathrm{E}$ & 23 & 23 & $0 \%$ & $69.7 \%$ \\
\hline $\mathrm{F}$ & 20 & 19 & $5 \%$ & $57.6 \%$ \\
\hline $\mathrm{G}$ & 18 & 17 & $5.6 \%$ & $51.5 \%$ \\
\hline $\mathrm{H}$ & 21 & 19 & $9.5 \%$ & $57.6 \%$ \\
\hline
\end{tabular}

Table 4 shows a comparison of the operation codes needed by each OpenCL kernel with the other kernels. In this case, each operation code needed in the optimized version of code is considered an entry in a vector. Each kernel's vector is compared as a Hamming distance to the other kernel. The table shows the difference in operation codes necessary for the MicroBlaze processor implementation. Some of the highlights include that some kernels (A \& D: Bitonic Sort and Histogram) require the identical operation codes. In this case, the processor operation code design and internal data path would be identical. In the extreme case, kernel E (Mandelbrot) has the largest distinction in that it varies with the other kernels by a minimum of 10 operation codes and most widely with the other kernels by 14 operation codes. These results indicate that a set of soft processors may be dedicated to set of algorithms identified by their OpenCL kernels.

Table 4. Hamming Analysis of Required Kernel Opcode Set

\begin{tabular}{|l|r|r|r|r|r|r|r|r|}
\hline Kernel & \multicolumn{1}{|c|}{ A } & \multicolumn{1}{c|}{ B } & \multicolumn{1}{l|}{ C } & \multicolumn{1}{l|}{ D } & E & F & G & H \\
\hline A & - & 6 & 2 & 0 & 14 & 6 & 4 & 6 \\
\hline B & 6 & - & 8 & 6 & 12 & 10 & 6 & 10 \\
\hline C & 2 & 8 & - & 2 & 12 & 4 & 4 & 6 \\
\hline D & 0 & 6 & 2 & - & 14 & 6 & 4 & 6 \\
\hline E & 14 & 12 & 12 & 14 & - & 10 & 10 & 12 \\
\hline F & 6 & 10 & 4 & 6 & 10 & - & 6 & 8 \\
\hline G & 4 & 6 & 4 & 4 & 10 & 6 & - & 8 \\
\hline H & 6 & 10 & 6 & 6 & 12 & 8 & 8 & - \\
\hline
\end{tabular}

\section{EXPERIMENTAL FINDINGS}

\subsection{Methodology}

We targeted synthesis of the OpenCL kernels to various FlexCL organizations for a Xilinx Virtex5 SX95T using the Xilinx Design tools. Since most of the FPGA is based on Look-up-Tables (LUTs), this was considered the best resource target. In addition, there are FPGA registers, Block RAMs (BRAMs), and DSPs. Overall, the elementary programmable logic block in Xilinx FPGAs is called a slice. As such, the typical Xilinx slice includes the overall area for multiple LUTs, dedicated multiplexors, dedicated arithmetic logic, and registers.

\subsection{FPGA Synthesis Results}

We evaluated several designs of MicroBlaze-based MPSoCs. Specifically we explored fitting as many cores as possible on the 
target Xilinx XC5VSX95T. The results of Figure 10 show the percentage of the FPGA's resources (registers, LUTs, BRAMs, DSPs) for several targets is subject to variations in 4 primary design attributes impacting OpenCL targets: number of cores, local memory, number of local memory banks, and number of floating-point units. In these experiments, we performed the full implementation of the FlexCL core (no feature customization) with two goals: (1) study the potential of placing as many cores as possible on the FPGA and (2) build an exploration table containing FPGA resource estimates of each FlexCore processor. The estimates relate each large configuration component (core, local memory bank, local memory space, and FPU) to the total number of FPGA LUTs and slices. Overall, the results indicate that as many as 16 FlexCL cores could be places on an FPGA MPSoC. Local memory and local memory banking are studied in Figure 10, showing that doubling the banking support (necessary for simultaneous workgroup access) typically requires about $5 \%$ of the slice area when all other parameters are maintained constant.

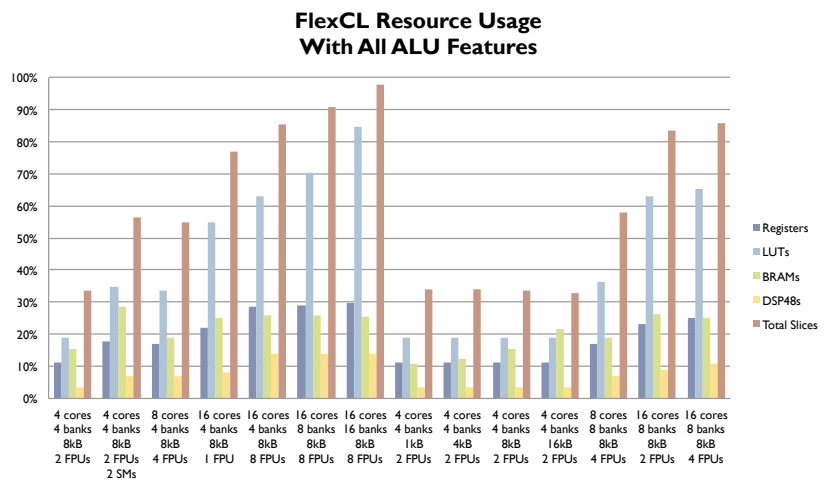

Figure 10 FlexCL FPGA Resource Utilization

Figure 11 includes the impact of custom FPGA designs for FlexCL processors with various processor datapath and OpenCL support configurations. The base design of Figure 11 is an 8-core OpenCL compute unit, 8-banked local memory (8KB) with 4 FPUs. The largest effects of individual changes in the datapath and/or opcode requirements come from the elimination of requirements of barrel-shifting and FPU division from the core processor configuration. In these cases there are respective savings of $14 \%$ and $6 \%$ of FPGA Look-up-Table (LUT) savings in FPGA space. While these two features are clearly dedicated for use in certain kernels, there are other architecture features that are based on more detailed analysis. For example, the register forwarding support can save only 5\% FPGA space for a FlexCL core, but only a few kernels require register forwarding (passing of registers between the two nearest instructions in the pipeline). Similarly, related to OpenCL support, the conditional and unconditional support provides insight into the branch divergence support. In this case, detecting whether branch divergence is possible within the workgroup of a compute unit, will largely be based on the branching support, which amounts to $2 \%$ of core area.

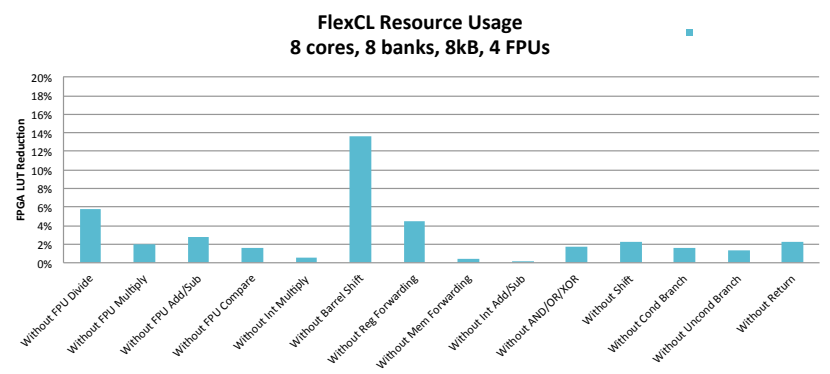

Figure 11 FlexCL FPGA Utilization vs. Features (Datapath \& OpenCL Configurations)

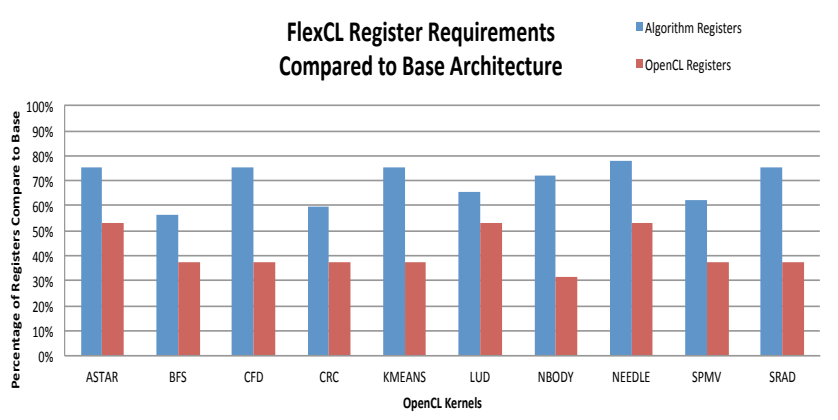

Figure 12 FlexCL Register Architecture Evaluation

Figure 12 shows the analysis of the registers necessary for a FlexCL architecture for a set of substantially larger OpenCL kernels than studied earlier. The OpenDwarfs [12] project provides an OpenCL benchmark suite consisting of different computation/communication idioms (i.e. dwarfs, for state-of-art multicore and GPU systems). The OpenDwarfs are based on the Berkeley Dwarfs [14], which described a dwarf as an algorithmic method that captures a pattern of numerical computation and communication believed to be important for science and engineering. These benchmarks consist of A* (ASTAR), Breadth First Search (BFS), Computational Fluid Dynamics (CFD), Cyclic Redundancy Calculation (CRC), K-means clustering (KMEANS), lower-upper decomposition (LUD), N-body simulation (NBODY), Needleman-Wunsch Needleman-Wunsch (NEEDLE) DNA processing, Sparse Matrix-Vector Multiplication (SPMV), and Speckle Reducing Anisotropic Diffusion (SRAD). The results compare the analysis of OpenCL kernels to the requirements of a full Microblaze-based 32-register file architecture combined with the OpenCL built-in registers. By customizing the architecture to the OpenCL requirements of the kernel, each benchmark can lower the number of registers required for the algorithm and for the OpenCL implementation. On average, only $60 \%$ of the full $32-$ entry register file are required to carry out each benchmark, and only $40 \%$ of the OpenCL registers are necessary if the FlexCL architecture register design is deployed. These reductions directly relate to the space exploration by eliminating unnecessary FPGA space for registers from the architecture description. The results indicate an important concept in design space exploration: that to maximize the number of cores per chip, the characteristics of the targeted application set must involved in the process. 


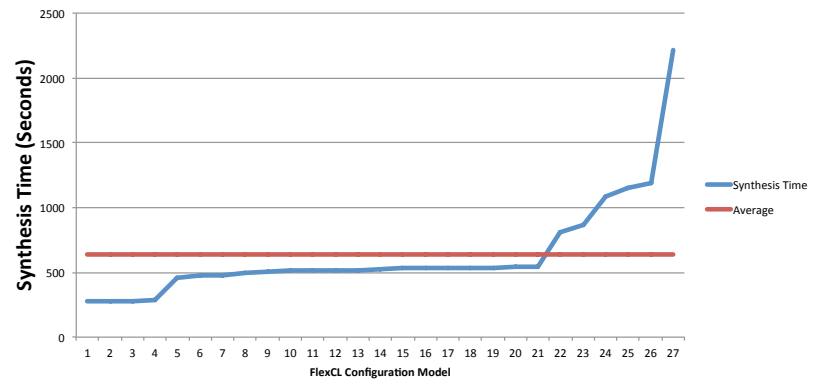

Figure 13 FlexCL Exploration Synthesis Time

Figure 13 shows the synthesis time in seconds to configure the 28 designs used in the current FlexCL study. The average line indicates the average of the 28 synthesized designs, which is 635 seconds. The base design of is an 8-core OpenCL compute unit, with 8-banked local memory (8KB) and 4 FPUs. The synthesis times include both the datapath and OpenCL configurations. Out of the sorted synthesis times, the very last point is the full design without any modification. Overall, for understanding how a design space exploration might be developed within the FlexCL framework, it would be expected that each design would require 10 minutes (approximately 600 seconds). While the synthesis times are not significant, depending on the number of design alternatives, it may be necessary to cull the space by using additional pre-synthesis estimates on performance.

\section{CONCLUSIONS AND FUTURE WORK}

An OpenCL kernel represents the execution of parallel resources in a system and there has been significant interest in the image processing, computer vision, computational photography, continuous sensing, and 3D processing fields. Running counter to the FPGA model is momentum of a software community pressing the development of a growing set of OpenCL kernels exploitable by CPU/GPU architectures supporting OpenCL.

Overall, we believe the use of custom OpenCL designs for FPGAs offers several potential advantages in comparison to traditional FPGA-based MPSoC methodologies. Such systems have the advantage of receiving both software (compiler) and hardware (synthesis) optimizations. The software techniques are beneficial as they can be applied after design release, which is not always feasible with hardware-based systems. Similarly, it may be possible to steer the compiler to generate code sequences that use a small set of FPGA synthesizable resources, thus making space for more processing elements. Other target FPGA parts will be considered to provide more detailed synthesis results, as well as more detailed comparison of synthesis of resources achievable when strategically eliminating certain combinations of features.

\section{REFERENCES}

[1] The OpenCL Specification Version: 1.1 - Khronos OpenCL Working Group Editor: Aaftab Munshi

[2] Xilinx Zynq: http://www.xilinx.com/products/silicondevices/soc/zynq-7000/index.html
[3] John Nickolls, Ian Buck, Michael Garland, and Kevin Skadron. 2008. Scalable Parallel Programming with CUDA. Queue 6, 2 (March 2008), pp. 40-53.

[4] C. Jalier, et al. "Heterogeneous vs Homogeneous MPSoC Approaches for a Mobile LTE Modem". In: DATE, 2010, pp. 184-189.

[5] Ahsan Shabbir, Akash Kumar, Bart Mesman, Henk Corporaal: Enabling MPSoC Design Space Exploration on FPGAs. IMTIC 2008, pp. 412-421.

[6] S. Ahmed et al., "Design of heterogeneous MPSoC on FPGA”. In: ASIC, 2007, pp. 102-105. http://doi.ieeecomputersociety.org/10.1109/FPL.2010.6

[7] Göhringer et al. Reconfigurable MPSoC versus GPU: Performance, Power and Energy Evaluation. In Proceedings of the 2011 International Conference on Industrial Informatics (INDIN), Lisbon, Portugal.

[8] Valle, Del; Pablo, et al, Architectural Exploration of MPSoC Designs Based on an FPGA Emulation Framework Proceedings of 2006 Conference on Design of Circuits and Integrated Systems (DCIS), pp. 12-18.

[9] MicroBlaze Processor Reference Guide - Embedded Development Kit EDK 10.1i

[10] "The LLVM Compiler Infrastructure Project.” [Online]. Available: http://llvm.org/.

[11] Tianyi David Han et al. 2011. Reducing branch divergence in GPU programs. In Proceedings of the Fourth GPGPU Workshop ACM, New York, NY, USA, Article 3.

[12] "OpenCL and the 13 Dwarfs: A Work in Progress" by Feng, Lin, Scogland, and Zhang in the 3rd ACM/SPEC International Conference on Performance Engineering, 2012

[13] "The Partial Reverse If-Conversion Framework for Balancing Control Flow and Predication," David August, Wen-mei Hwu, and Scott Mahlke, International Journal of Parallel Programming, Vol. 27, Oct. 1999, pp. 381-423.

[14] Asanovic et al., A view of the parallel computing landscape. Commun. ACM 52, 10 (October 2009), pp. 56-67.

[15] "OpenCL for Altera FPGAs: Accelerating Performance and Design Productivity" [Online]. Available: http://www.altera.com/opencl

[16] Lebedev et al., MARC: A Many-Core Approach to Reconfigurable Computing. Proceedings of 2010 International Conference on Reconfigurable Computing and FPGAs (ReConFig), December 2010, pp. 7-12

[17] Diana Göhringer, Jürgen Becker. 2010. High Performance Reconfigurable Multi-Processor-Based Computing on FPGAs. Proceedings of 2010 IEEE International Symposium on Parallel \& Distributed Processing, Workshops and Phd Forum (IPDPSW), April 2010 